$\begin{array}{cl}\begin{array}{cl}\text { Revue } \\ \text { de /histoire }\end{array} & \text { Revue de l'histoire des religions } \\ \text { des religions } & \begin{array}{l}\mathbf{3} \text { |2008 } \\ \text { Varia }\end{array}\end{array}$

\title{
Mary Ward et sa Compagnie de Jésus au féminin dans l'Angleterre de la Contre-Réforme
}

A Society of Jesus for Women? The Controversy about Mary Ward's Project in Early Modern England

Laurence Lux-Sterritt

\section{OpenEdition}

Journals

Édition électronique

URL : http://journals.openedition.org/rhr/6873

DOI : $10.4000 /$ rhr.6873

ISSN : 2105-2573

Éditeur

Armand Colin

Édition imprimée

Date de publication : 1 septembre 2008

Pagination : 393-414

ISBN : 978-2200-92445-4

ISSN : 0035-1423

\section{Référence électronique}

Laurence Lux-Sterritt, « Mary Ward et sa Compagnie de Jésus au féminin dans l'Angleterre de la Contre-Réforme », Revue de l'histoire des religions [En ligne], 3 | 2008, mis en ligne le 01 septembre 2011, consulté le 14 novembre 2019. URL : http://journals.openedition.org/rhr/6873 ; DOI : 10.4000/ rhr.6873 


\section{Mary Ward et sa Compagnie de Jésus au féminin dans l'Angleterre de la Contre-Réforme}

Insolite reflet de la querelle des femmes qui occupe les érudits, la polémique cléricale autour de l'Institut catholique fondé en 1611 par Mary Ward (1585-1645) tente de déterminer si ces étranges religieuses, qui se veulent missionnaires, sont les émissaires de Dieu ou les agents du mal. Tandis que leurs amis louent leur vertu, leurs nombreux ennemis contestent la probité de ces femmes qu'ils pensent corrompues. Leur vocation ne leur semble pas divine, mais maléfique, et l'innovation d'une Compagnie de Jésus au féminin, usurpant l'autorité cléricale, est la preuve de leur déviance. Convaincu, Urbain VIII éradique l'Institut en 1631 et le déclare nuisible à l'Église.

\section{A Society of Jesus for Women? The Controversy about Mary Ward's Project in Early Modern England}

Peculiarly echoing the ongoing "querelle des femmes", the clerical polemic which developed around the catholic Institute founded in 1611 by Mary Ward (1585-1645) pondered whether these unusual missionary nuns could in fact be considered as Soldiers of God. Whereas their friends hailed them as saints and mystics of angelic virtue, their many enemies questioned the probity of these women, whom they viewed, by their very nature, as corrupted. Their novel vocation seemed evil rather than divinely inspired, and their deviance was believed to be illustrated in their Society of Jesus for women, which usurped clerical authority. In 1631, these arguments convinced Urban VIII, who eradicated the Institute, declaring it harmful to the Church. 
Le 5 novembre est jour de fête nationale en Angleterre : les feux de joie que l'on allume, et sur lesquels on brûle des effigies du catholique Guy Fawkes, sont l'occasion de commémorer le fameux complot des poudres découvert le 5 novembre 1605. Ainsi, l'histoire du pays ne peut faire l'économie d'une histoire de l'anticatholicisme profond qui le façonne. Depuis le schisme du roi Henri VIII, qui déclare l'Angleterre indépendante de l'Église de Rome en 1533 et se fait proclamer unique chef de l'Église d'Angleterre en 1534, le royaume construit son identité nationale en utilisant le catholicisme comme puissant repoussoir ${ }^{1}$. Dans l'Angleterre protestante du dixseptième siècle les catholiques, que l'on appelle aussi « récusants » quand ils refusent de se conformer à l'église établie, sont soumis à des lois très sévères. Dès 1581, le parlement d'Elisabeth I (15581603) déclare que c'est trahison de reconnaître l'autorité spirituelle du Pape, d'entendre la messe et d'aider les catholiques sur le sol anglais. En 1585, le Parlement concentre ses efforts sur les organisateurs de réseaux résistants et surtout sur les missionnaires formés sur le continent et qui, revenus clandestinement au pays, travaillent chaque jour à la reconversion du royaume ${ }^{2}$.

Dans ce contexte répressif se développe pourtant une tentative de survie, et même de renouveau, au sein de la minorité catholique ; inspiré par l'esprit de la Contre-réforme, un nombre croissant de familles envoie ses fils aux séminaires de Douai, de Reims ou de Rome. Au péril de leur vie, ces jeunes missionnaires reviendront ensuite sur leur sol natal pour y porter assistance à leurs coreligionnaires et tenter de convertir les autres. C'est dans cette atmosphère que Mary Ward (1585-1645) grandit dans le Yorkshire avant de partir

1. En 1533, l'acte dit « in Restraint of Appeals to Rome» déclare que le royaume d'Angleterre est un empire indépendant de l'autorité du pape, que l'on désigne désormais sous le titre de simple évêque de Rome; en 1534, avec l'Acte de Suprématie, nait l'Anglicana Ecclesia, dont le seul chef suprême n'est autre que le roi.

2. 1581, 23: Elis. I, c.1, Act to Retain the Queen's Majesty's Subjects in their Due Obedience; et 1585, 27: Elis. I, c.2, Act against Jesuits, Seminary Priests and such other like Disobedient Persons. 
pour Saint-Omer, où elle devient clarisse en $1606^{3}$. Or, le cloître ne lui procure pas la sérénité qu'elle espérait y trouver; plus tard, tandis qu'elle écrit quelques bribes d'autobiographie, elle se souvient des années 1607 à 1611 comme d'une période difficile. En 1609, elle obtient la permission d'ouvrir un nouvel établissement de clarisses, pour y regrouper toutes les postulantes anglaises. Mais son mal-être demeure, et c'est seulement en 1611 qu'elle trouve enfin sa vocation. Dans sa correspondance, elle décrira plus tard avoir reçu l'ordre divin, «Take the Same of the Society»; elle pense donc comprendre que Dieu souhaite qu'elle se consacre à un apostolat comparable à celui de la Compagnie de Jésus ${ }^{4}$. En 1612, elle quitte son Ordre pour fonder à Saint-Omer une maison de « Dames anglaises » qui, dès l'abord, se considèrent comme religieuses bien que n'étant pas reconnues par le Pape.

La formule est un succès et Mary Ward ouvre d'autres établissements à Liège (1616), à Cologne et à Trier (1620-1621), à Rome (1622), à Naples et à Pérouse (1623), à Munich et à Vienne (1627) et enfin à Presbourg et à Prague (1628). Ces maisons accomplissent des tâches diverses. Dans leurs internats, de jeunes Anglaises issues de familles aisées achèvent leur éducation catholique loin des dangers de leur terre natale. Leurs externats, eux, accueillent des élèves de condition humble, souvent issues de la population locale. Enfin, les instituts fonctionnent aussi comme des séminaires où les Dames anglaises sont formées aux devoirs de leur mission apostolique.

3. Voir la biographie de M.C.E. Chambers, The Life of Mary Ward, 15851645, 2 vols, Londres, 1882-1885 et la remarquable histoire de l'Institut écrite par Henriette Peters, Mary Ward: A World in Contemplation, traduit de l'allemand par Helen Butterworth, Leominster, 1994. Voir aussi Laurence LuxSterritt, Redefining Female Religious Life: French Ursulines and English Ladies in Seventeenth-Century Catholicism, Aldershot, 2005.

4. Archives du Bar Convent, York (ABC), B5, lettre 4 à Mgr Albergati, 1620. Le Bar Convent, couvent fondé à York par les consœurs de Mary Ward en 1686, est aujourd'hui encore actif; c'est le plus ancien couvent du royaume. Il conserve dans ses archives des copies des documents concernant l'Institut durant la vie de Mary Ward, dont les originaux se trouvent au généralat de Munich. Il préserve aussi tous les originaux ayant trait à ses propres membres et aux autres centres clandestins fondés en Angleterre pendant l'ère moderne. Traduction de l'anglais par mes soins. 
Pour secourir les récusants et convertir les protestants d'Angleterre, Mary Ward crée également des centres clandestins dans le royaume et développe un réseau qui s'étend bientôt de Londres jusqu'aux régions du nord du Yorkshire.

Depuis une dizaine d'années, des auteurs comme Frances Dolan, Arthur Marotti, Michael Questier et Peter Lake, Alison Shell, Raymond Tumbleson ou Alexandra Walsham ont publié d'excellentes études sur la représentation du catholique dans l'imaginaire collectif anglican ${ }^{5}$. Ces études se penchent naturellement sur les traités des défenseurs de l'anglicité, protestants convaincus qui dénoncent les «papistes » et particulièrement les jésuites, comme source de tous les maux. Or, les fils spirituels d'Ignace de Loyola sont l'objet d'une violente dispute au sein même de l'Église catholique et la voix du clergé séculier rejoint parfois étrangement celle des protestants pour formuler les mêmes reproches à l'encontre de la Compagnie de Jésus. Dans un contexte religieux si agité, la communauté de Mary Ward, calquée sur le modèle jésuite, se retrouve attaquée de toutes parts, par les polémistes anticatholiques, bien sûr, mais aussi par le clergé séculier et même par les jésuites eux-mêmes. La controverse qui fait rage dans les années 1620 se conclut en 1631 quand le pape Urbain VIII condamne l'Institut à l'abolition perpétuelle dans sa bulle Pastoralis Romani Pontificis ${ }^{6}$.

5. Voir Frances Dolan, Whores of Babylon. Catholicism, Gender and Seventeenth Century Print Culture, Ithaca, 1999 ; Peter Lake, «Anti-Popery: the Structure of a Prejudice », in Richard Cust et Ann Hughes (dir.), Conflict in Early Stuart England, Londres, 1989 ; Peter Lake et Michael Questier, The Antichrist's Lewd Hat: Protestants, Papists and Players in Post-Reformation England, New Haven, Conn., 2002 ; Arthur Marotti (dir.), Catholicism and Anti-Catholicism in Early Modern English Texts, Basingstoke, 1999, et Religious Ideology and Cultural Fantasy. Catholic and Anti-Catholic Discourses in Early Modern England, Notre Dame, Ind., 2005 ; Alison Shell, Catholicism, Controversy and the English Literary Imagination, 158-1660, Cambridge, 2006 ; Raymond Tumbleson, Catholicism in the English Protestant Imagination. Nationalism, Religion, and Literature, 1600-1745, Cambridge, 1998 et Alexandra Walsham, Church Papists: Catholicism, Conformity and Confessional Polemic in Early Modern England, Woodbridge, 1993.

6. Voir Immolata Wetter, Mary Ward. Under the Shadow of the Inquisition, Oxford, 2006 et Laurence Lux-Sterritt, « An Analysis of the Controversy Caused by Mary Ward's Institute in the 1620s », Recusant History 25.4 (2001), 636-47. 
Les facteurs qui poussent Urbain VIII à prononcer la suppression de l'Institut sont trop complexes pour être envisagés ici en détail. Il suffira de nous pencher sur les principaux aspects de la vocation des Dames anglaises pour y percevoir des éléments qui, à eux seuls, auraient amené la condamnation de celles qui se voulaient être les « soldats de Dieu ${ }^{7}$. Leur Compagnie de Jésus au féminin est officiellement rejetée comme une aberration, puisque Ignace de Loyola interdit formellement sa Compagnie aux femmes et que le Concile de Trente (1545-63) proscrit la création de nouveaux ordres. De plus une communauté de religieuses faisant fi des règles de la clôture irait à l'encontre de la loi canon et de la morale chrétienne : l'Institut envisagé par Mary Ward ne saurait donc exister, ne pouvant être à la fois régulier et apostolique. La controverse repose pourtant sur un paradoxe évident: alors même que le clergé démontre qu'il est impossible de reconnaître une Compagnie de Jésus au féminin, c'est néanmoins en tant que « jésuitesses » que les Dames anglaises subissent les pires attaques : afin d'éliminer une communauté si proche de ses rivaux réguliers, le clergé séculier n'hésite pas à emprunter aux polémistes protestants les arguments les plus éprouvés de la diatribe anti-jésuite.

\section{La Vocation ignaCienNe de Mary Ward}

Dès l'époque moderne, les recensements officiels (par exemple les recusant rolls) montrent une surreprésentation des femmes dans la communauté récusante, que l'historien John Bossy voit d'ailleurs comme un «matriarcat $»^{8}$. Cette prépondérance féminine peut être expliquée par divers facteurs, mais dans le cas des épouses d'hommes déclarés conformistes Alexandra Walsham a démontré qu'il s'agissait souvent de pactes passés entre conjoints pour préserver le patrimoine familial en évitant les lourdes amendes ou les confiscations de biens qui sanctionnent la récusance ${ }^{9}$. La femme mariée est en effet protégée par son statut de feme covert: ne possédant aucun capital en son nom propre, elle n'est que marginalement atteinte par

7. ABC, B18, Institutum, f. 19.

8. John Bossy, The English Catholic Community, 1570-1850, Londres, 1976.

9. Walsham, Church Papists, pp.78-81. 
la loi et jouit donc d'une liberté religieuse que de nombreux foyers sauront mettre à profit. Elle sera récusante et assurera l'éducation de leurs enfants et la pratique de la foi au quotidien, tandis qu'il deviendra Church-papist, évitant les poursuites par sa conformité publique ${ }^{10}$. L'État anglais, sans le vouloir, semble avoir contribué à donner aux femmes une véritable importance dans l'organisation de la communauté catholique clandestine, et ce sont elles que les missionnaires citent souvent dans leurs récits pour leurs qualités d'hôtesses et de collaboratrices.

L'entreprise de Mary Ward cherche à permettre aux femmes de transcender le statut de simples « aides » de la mission, pour faire d'elles de véritables agents. Ses Dames anglaises se considèrent comme religieuses mais préfèrent à la clôture traditionnelle la liberté de mouvement nécessaire à toute entreprise missionnaire, au même titre que les jésuites. Cette vocation répond à un besoin criant en Angleterre où, faute de couvents, les femmes qui souhaitent entrer en religion se voient de facto condamnées à l'exil, tandis que celles qui demeurent dans le siècle ne bénéficient guère d'une mission qui s'adresse d'abord aux hommes. Les Dames anglaises pallient ce manque en adoptant le modèle typique des clercs réguliers de la Contre-Réforme pour apporter le soutien spirituel nécessaire à leurs coreligionnaires en Angleterre et ailleurs.

Après 1611, année où elle déclare avoir reçu l'ordre divin d'imiter la Compagnie de Jésus, la fondatrice tente à plusieurs reprises d'obtenir l'approbation papale. Dès 1612, aidée par le jésuite Roger Lee, son confesseur, elle ébauche un premier plan, intitulé Schola Beatae Mariae, dans lequel elle présente la raison d'être de son Institut ${ }^{11}$.

10. L'adjectif péjoratif «papiste » est utilisé en règle générale par les protestants dénonçant, par ce sobriquet, tous les catholiques. Au contraire, le terme « récusant » est un terme légal se rapportant à toute personne refusant de se présenter au service dominical anglican. Par glissement, le terme fut employé pour définir les catholiques refusant ouvertement de se conformer. Les « récusants » se différencient des Church papists qui, souvent pour échapper aux sanctions, assistent à l'office bien qu'étant catholiques en privé.

11. Les documents concernant les demandes d'approbation de l'Institut sont conservés au généralat de Munich; des copies sont consultables aux Archives du Bar Convent (ABC) de York dans la liasse B18. Ici, voir ABC, B18, Schola Beatae Mariae, item 1. 
Elle rappelle aux autorités romaines les circonstances particulières de son Angleterre natale, et les conditions difficiles dans lesquelles la minorité catholique tente de survivre. Elle ose suggérer que, dans les pays protestants, le modèle tridentin de la religieuse cloîtrée n'est pas le mieux adapté ; sans nier l'utilité des prières offertes par ces communautés, elle souhaite néanmoins obtenir l'autorisation d'engager des religieuses dans une vie dite «mixte», mariant contemplation et action afin de compléter l'effort des missionnaires masculins.

En 1616, la fondatrice soumet un deuxième Plan (Ratio Instituti), soulignant une fois encore la dimension pragmatique de son Institut :

Puisque l'Angleterre, tristement affligée [...] se trouve dans un si grand besoin de travailleurs spirituels, et puisque les prêtres [...] travaillent assidûment comme apôtres dans cette moisson, il semble que le sexe féminin lui aussi, dans la mesure de ses moyens, doive et puisse, d'une façon semblable, contribuer plus qu'à l'ordinaire face à ce besoin spirituel commun ${ }^{12}$.

Elle l'écrit sans détour: les femmes ont le devoir moral de s'impliquer dans cette crise, et elles ont les capacités physiques et spirituelles de travailler dans l'apostolat comme le font les missionnaires.

Il faut plusieurs années avant que la nature précise de sa vocation ignacienne ne soit formulée clairement avec un troisième et dernier Plan, la Ratio Instituti de 1621. Très largement inspiré des Formula Instituti de la Compagnie de Jésus, ce document adapte les constitutions jésuites pour l'usage des Dames anglaises, et Mary Ward demande au pape Grégoire XV d'approuver ce qu'elle nomme sans détour sa Compagnie de Jésus au féminin:

Quiconque désire servir sous la bannière de la croix comme soldat de Dieu dans notre Compagnie, que nous voulons désigner sous le nom de Jésus, [...] est membre d'une Compagnie fondée avant tout pour la défense et la propagation de la foi et pour aider les âmes à progresser dans un mode de vie chrétien et à mieux connaitre la doctrine, pour les détourner de l'hérésie et du mal ${ }^{13}$.

12. ABC, B18, Ratio Instituti, f. 1.

13. ABC, B18, Institutum, f. 18-9 (mes italiques). 
Elle accompagne ce Plan d'une brève déclaration (Brevis Declaratio) dans laquelle elle justifie sa vocation et son imitation des jésuites :

Nous ne pouvons que constater et regretter que ce qui est approuvé pour l'éducation prodiguée aux garçons par la Compagnie de Jésus n'existe pas pour les filles; il en résulte que la moitié de la race humaine $[\ldots]$ semble, sinon tout à fait laissée à elle-même, du moins ne pas recevoir grande assistance ${ }^{14}$.

Si la fin de l'Institut se veut en tous points similaire à celle des jésuites, il en va de même pour sa structure, chaque branche régionale étant administrée par une supérieure, elle-même soumise à l'autorité unique d'une supérieure générale, Mary Ward elle-même. Tout comme la Compagnie de Jésus, l'Institut se soustrait à l'autorité traditionnelle des évêques et se veut entièrement autonome : c'est la générale qui décide des actions à mener et des moyens à mettre en œuvre, et il n'est d'autorité supérieure à la sienne que celle du Saint-Père lui-même. Ainsi, dans l'Institutum, les Dames anglaises demandent l'autorisation de prononcer, comme le font les jésuites, un vœu d'obéissance directe au pape ${ }^{15}$.

L'initiative novatrice de la récusante Mary Ward se veut donc à la fois religieuse et apostolique, et elle adapte fidèlement les règles de la Compagnie d'Ignace de Loyola, ses constitutions et jusqu'à son organisation hiérarchique. Or, si le clergé reconnaît volontiers que la mission anglaise manque cruellement d'entreprises apostoliques et enseignantes adressées aux femmes, c'est la forme choisie par la fondatrice qui semble constituer un obstacle infranchissable. Dans le contexte de l'Europe post-tridentine, les Dames anglaises enfreignent la loi canon et compromettent les valeurs de l'Église de la ContreRéforme : elles se heurtent donc sans tarder à de grandes difficultés, puisque leur Compagnie de Jésus pour femmes apparait à la grande majorité du clergé comme une monstruosité.

14. ABC, B 18, Brevis Declaratio.

15. ABC, B18, Institutum, f. 22. 


\section{Une CoMpagnie de JÉSUS AU FÉMININ : UNE AbERRATION CANONIQUE ET MORALE}

Une aberration, en effet, car les femmes sont exclues de la Compagnie de Jésus par son fondateur, Ignace de Loyola (14911556); les Dames anglaises ne peuvent donc en aucun cas être considérées comme faisant partie des jésuites, ni comme leur étant affiliées. En outre, la création d'une communauté de religieuses imitant le modus vivendi des missionnaires n'est pas envisageable, puisque le Concile de Trente a non seulement interdit la création de nouveaux Ordres, réitérant les décrets du quatrième concile œcuménique de Latran (1215), mais aussi ordonné la plus stricte clôture pour les Ordres religieux féminins, renforçant à cette occasion la décrétale Periculoso (1298) de Boniface VIII ${ }^{16}$. Un Ordre de religieuses doit par conséquent être cloîtré et adopter l'une des règles monastiques déjà existantes. Ces principes de la Réforme catholique sont défendus avec d'autant plus de véhémence qu'ils sont souvent mis en péril au dix-septième siècle, ère où fleurissent de nouvelles congrégations à vocation apostolique. La dialectique de l'action et de la contemplation est l'un des sujets les plus brûlants de l'époque, et le Français Jean-Baptiste Thiers, dans son Traité de la clôture des religieuses, déclare qu'il n'y a chez les religieuses « rien qui soit plus contraire à l'esprit de la Religion que le violement de la clôture »; il se fait le porte-parole du clergé quand il écrit que « les religieuses qui sortent de leurs monastères sont dans un état scandaleux et injurieux à Dieu et à la religion ${ }^{17}$.

La mobilité géographique de l'Institut de Mary Ward devient très vite une pierre d'achoppement majeure ; l'idée d'une communauté missionnaire féminine est tournée en dérision, tant il est impensable que des religieuses s'éloignent du modèle conventuel. Les Dames anglaises qui travaillent incognito en Angleterre se voient alors affublées de sobriquets comme les « commères errantes », les «nonnes

16. Norman Tanner (dir.), Decrees of the Ecumenical Councils, Londres, 1990, vol. 1, p. 227-73.

17. Jean-Baptiste Thiers, Traité de la clôture de religieuses, Paris, 1681, p. 254. 
errantes », ou les "galopeuses » ${ }^{18}$. Il est vrai qu'elles changent fréquemment de lieu de résidence, usent de pseudonymes et jouent beaucoup du déguisement pour échapper aux sanctions du gouvernement : vêtues tantôt comme de nobles dames, tantôt comme de pauvres femmes, elles changent d'identité selon la circonstance pour passer inaperçues ${ }^{19}$. En 1621, dix représentants du clergé séculier rédigent un virulent mémoire contre l'Institutum présenté au pape par la fondatrice. Sous la direction de l'archiprêtre William Harrison $(\dagger 1621)$, ils signent l'Informatio pour protester contre un Institut qui leur semble « en contradiction directe avec les décrets du Concile sacré de Trente ${ }^{20}$.

Même s'il reconnaît le travail de catéchistes et d'enseignantes des Dames anglaises comme digne de respect, le clergé séculier s'entend pour condamner le modus operandi choisi par Mary Ward. En octobre 1622, le prêtre John Colleton (1548-1635) souligne à nouveau l'infraction à la clôture desdites « jésuitesses » :

Si [l'Institut] se cantonnait à ses cellules et à ses murs, suivant l'exemple d'autres communautés religieuses, [il] serait peut-être louable ; mais quand il s'arroge les devoirs de l'office apostolique, erre sans restriction ici et là, [...] entreprend la direction spirituelle des familles et bien d'autres choses encore sous prétexte de charité chrétienne, et qu'en dépit de tout cela [il] insiste pour être compté parmi les rangs des communautés religieuses, [il] s'expose certainement à la censure et aux reproches de bien des gens de piété ${ }^{21}$.

Cette condamnation de la mobilité géographique des Dames anglaises est d'ailleurs l'un des rares sujets de consensus entre le

18. Lewis Owen, Running Register, Londres, 1626.

19. La première biographie de Mary Ward, A Briefe Relation of the Holy Life and Happy Death of our Dearest Mother, est écrite par ses consœurs Mary Poyntz et Winifred Wigmore autour de 1650. Le Bar Convent de York en conserve des photographies (liasse A11) et une transcription (liasse A12). Ici, voir A12, f. 20.

20. Archives du diocèse de Westminster (ADW), vol. 16 (1617-22), ff. 201-07, Copia Informationis de Jesuitissis.

21. ABC, C1, J3, translation of documents re. Jesuitesses. John Colleton (1548-1635), fondateur de l'Association du clergé d'Angleterre chargée des affaires du clergé séculier, sera suspendu pour avoir envenimé la dispute entre séculiers et jésuites. Voir Ethelred Taunton, The History of the Jesuits in England, Londres, 1901, pp. 256-57. 
clergé séculier et le clergé régulier à cette époque. La polémique qui, dès le début du dix-septième siècle, oppose en Europe les jésuites aux séculiers, est exacerbée dans le contexte de la mission anglaise, où les enjeux de pouvoir sont importants. Cependant, malgré le schisme grandissant entre les jésuites et les prêtres séculiers ${ }^{22}$, les deux partis s'accordent à condamner ensemble l'initiative de Mary Ward. Le jésuite canoniste Francisco Suarez (1548-1617) ajoute ainsi sa voix à celle de ses rivaux quand il s'oppose au mode de vie non cloitré des Dames anglaises qui, écrit-t-il, « vont et viennent dans tout le pays au mépris de l'étiquette convenant au sexe féminin et au grand déshonneur de la religion catholique ${ }^{23}$.

Mais le manque de clôture n'est pas la seule difficulté posée par la vocation novatrice des « jésuitesses ». Dans les mois qui suivent, de vives accusations se joignent à l'Informatio, pour demander la suppression de l'Institut. Une plainte est d'abord adressée au pape en mai 1622 par le Frère Robert Sherwood, bénédictin. Puis c'est Matthew Kellison, président du séminaire anglais de Douai, qui inclut dans son rapport sur le statut des séminaires en Flandres un procès-verbal contre l'Institut de Mary Ward ${ }^{24}$. Tandis que le clergé se défie de l'engagement croissant des femmes dans la ContreRéforme, les critiques de l'Institut soulignent ce qu'ils décrivent comme une incompatibilité essentielle entre féminité et mission : la femme, écrit-on, n'a ni la force physique, ni la rectitude morale, ni les capacités spirituelles nécessaires à une telle vocation. Selon

22. Voir entre autres le très célèbre plaidoyer de Christopher Bagshaw, A true relation of a faction begun at Wisbich by Fa. Edmonds, alias Weston, a Jesuite, 1595 and continued since by Fa. Walley, alias Garnet, the Provinciall of the Jesuits in England, and by Fa. Parsons in Rome, with their adherents : Against us the Secular Priests their brethren and fellow Prisoners, that disliked of novelties, and thought it dishonourable to the ancient Ecclesiastical Discipline of the Catholicke Church, that Secular Priests should be governed by Jesuits (presse anonyme, 1601).

23. ABC, C1, Letters against the Jesuitesses, f. 297.

24. Pour une analyse détaillée des documents écrits par Harrison, Sherwood et Kellison, voir Joseph Grisar, Die ersten Anklagen in Rom gegen das Institut Maria Wards 1622, Rome, 1959 et Ein schwieriger Rechtsfall zwischen den Englischen Fräulein und den englischen Jesuiten in Luttich 1616-1630, Rome, 1960. 
saint Paul, d'ailleurs, elle ne doit ni prêcher ni se mêler de discours religieux. Pour l'archiprêtre William Harrison, la propagation de la foi est l'affaire d'hommes alliant les enseignements du séminaire à une vertu inébranlable. Les Dames anglaises, au contraire, risquent d'enseigner dans leur catéchisme une doctrine erronée, ou d'être contaminées à tout moment par l'hérésie qui les entoure. Le contemporain de Mary Ward doute qu'une femme ait en elle la force de servir Dieu sans fléchir, et se plaît à rappeler le risque encouru par l'Église qui laisserait libre cours aux intentions apostoliques de filles qui, par stupidité si ce n'est par malice, finiraient par la desservir. Tous les détracteurs de l'Institut semblent d'accord sur ce point: une mission ne peut pas se concevoir pour les femmes, qui en sont physiquement, intellectuellement et moralement incapables.

Dans le contexte déjà tendu de l'époque, tout amalgame avec ces «nonnes errantes » prend donc une dimension particulièrement importante pour la Compagnie de Jésus, qui comprend que les accusations contre les Dames anglaises peuvent être utilisées également pour la discréditer. Les rapports initialement cordiaux entre Mary Ward et certains missionnaires se dégradent très vite, et un libelle anticatholique brocarde d'ailleurs le dilemme imposé aux jésuites par leurs plus ferventes adeptes :

[Les jésuites] sont en désaccord total vis-à-vis des jésuitesses, ou nonnes errantes ; certains les tolèrent, d'autres les ont en horreur. [Les Dames anglaises] portent l'habit ignacien et s'exposent à la vue de tous, accoutrées comme les jésuites ; elles diffèrent des autres nonnes en cela qu'elles vont et viennent dans le siècle et prêchent l'Evangile aux femmes en Angleterre et ailleurs ${ }^{25}$.

La Compagnie de Jésus est très embarrassée quand les Dames anglaises optent pour le port d'une robe noire évoquant leur habit ; elle prend alors ses distances avec l'Institut, auquel elle refuse d'être assimilée. Afin de combattre toute association erronée, les missionnaires reçoivent pour consigne de se dissocier publiquement de tout commerce avec les disciples de Mary Ward. La ligne de conduite officielle est énoncée par le général Muzio Vitelleschi (1563-1645) en 1623 , ordonnant à ses confrères de «ne se mêler d'aucune des

25. James Wadsworth, English Spanish Pilgrim, Londres, 1629, p. 30. 
affaires de Mary Ward ou des femmes de sa compagnie $»^{26}$. Les jésuites anglais qui ont par le passé soutenu la fondatrice, comme Roger Lee (1568-1615), John Gerard (1564-1637) ou Edward Burton (†1624) sont sévèrement réprimandés : il faut faire savoir à tous que la Compagnie de Jésus ne tolèrera aucune branche féminine de son Ordre et que ses membres n'entretiennent aucune relation avec lesdites «galopeuses ${ }^{27}$. En 1623, le rapport de mission de l'une des consœurs en mission en Angleterre évoque la dégradation des relations entre l'Institut et les jésuites sur le terrain ${ }^{28}$. Consciente de la mauvaise réputation dont pâtissent les Dames anglaises, sœur Dorothée cache sa véritable identité et son appartenance à l'Institut de Mary Ward à toutes ses connaissances, et même à ceux qu'elle convertit. À l'exception de Lady Timperley, qui la soutient, personne ne connait son secret. Alors qu'elle œuvre au sein de la communauté récusante, elle témoigne des conversations de laïcs mais aussi des propos d'un jésuite et d'un jeune homme se préparant à le devenir, reprenant à leur compte les accusations faites contre Mary Ward par les opposants de l'Institut.

En reniant l'Institut de Mary Ward, les jésuites cherchent à échapper à la calomnie qui prend ces femmes pour cibles ; mais plus essentiellement, en accord avec le clergé séculier, ils dénoncent le concept même de « jésuitesses ». La religieuse qui se veut apostolique outrepasse le rôle de la femme dans l'Église du dix-septième siècle, où la vie dite «mixte» reste la chasse gardée de l'homme. En 1617, un jésuite met en doute la constance des Dames anglaises confrontées aux rigueurs de la mission, arguant qu'elles ne sont après tout « que des femmes ». Face à la misogynie de son Église, Mary Ward s'insurge et déclare, lors d'un discours prononcé devant ses consœurs à Saint-Omer :

Rien n'est plus faux que d'affirmer que les femmes, à la différence des hommes, ne sauraient réaliser de grandes choses [...] Que pensez-vous

26. Lettre de Muzio Vitelleschi, le 19 juillet 1623 ; copie conservée aux $\mathrm{ABC}, \mathrm{C} 1, \mathrm{~J} 3$.

27. ADW, vol. 16, f. 207.

28. Le texte est transcrit dans M. C. E. Chambers, The Life of Mary Ward, pp. 226-32. 
de ce mot, « que des femmes » ? [...] les femmes, si elles s'y emploient, peuvent être parfaites, et si les hommes cessaient de nous assurer que nous ne pouvons rien accomplir et que nous ne sommes que des femmes, nous pourrions faire beaucoup ${ }^{29}$.

La fondatrice ne partage pas les idées communes à l'anthropologie de son temps, et sa revendication de la valeur féminine ne fait qu'exaspérer la hiérarchie ecclésiastique. Ainsi, pour Francesco Ingoli, secrétaire de la Propaganda Fide chargé de l'examen de l'Institut dans les années qui mèneront à sa suppression, la jeune femme cherche à effacer les différences entre les deux sexes, en se distançant de toutes les qualités de la féminité, mais aussi en usurpant celles de la masculinité. Ward est, selon lui, « une vierge à l'esprit viril » ${ }^{30}$, une « jeune femme à l'esprit masculin ${ }^{31}$. C'est cette rupture de communication avec les autorités romaines qui constitue l'un des obstacles les plus infranchissables pour l'Anglaise. Sa force de conviction, qui semble renier la différence essentielle entre l'homme et la femme, est perçue par le Saint-Siège comme un désir obstiné de transgresser les normes établies.

\section{L'INSTITUT CIBLE DE LA POLÉMIQUe ANTI-JÉSUITE}

Pragmatique, Mary Ward ne comprend pas que le pape n'accepte pas pour son Institut ce qu'il approuve pour la Compagnie de Jésus. Or, loin de faciliter la ratification de l'Institut, l'imitation du modèle jésuite s'avère en fait être rédhibitoire. Les jésuites sont en effet les missionnaires les plus honnis d'Angleterre, ce sont eux qui, dans les libelles protestants, incarnent la perfidie romaine et le renversement des valeurs anglicanes; ils sont considérés comme plus dangereux encore que les autres catholiques. Dans l'Anatomie of Popish Tyrannie, c'est donc le jésuite plus qu'aucun autre « papiste » qui représente le mal absolu :

29. ABC, B17, f. 2, Three Speeches of our Reverend Mother Chief Superior Made at St Omer Having Been Long Absent.

30. ABC, C1, Letters against the Jesuitesses, f. 312.

31. ABC, C18, f. 101. 
Notez bien, cher lecteur, que dépendre des jésuites c'est dépendre du diable; par conséquent, soutenir les jésuites, leurs desseins sanguinaires et leurs tragiques complots, n'est rien moins que renier Dieu, renoncer à le craindre et à le vénérer; c'est trahir votre prince ; c'est être l'ennemi de votre terre natale ${ }^{32}$.

Dans The Jesuits Downfall, Thomas James, polémiste anticatholique, s'en prend lui aussi aux jésuites comme étant les plus pernicieux de tous les missionnaires :

J'admets que nous devons craindre les prêtres aussi bien que les jésuites car ce sont là, à des degrés différents, de dangereux ennemis de sa Majesté et de l'État : mais les pires sont les jésuites, car ils réduisent leurs disciples à une servitude, voire à un esclavage tel que tout ce qu'ils disent a force de $\operatorname{loi}^{33} \ldots$

En Angleterre, l'anti-papisme qui règne depuis l'excommunication de la reine Elisabeth par le pape Pie V en 1570 atteint son paradoxisme au début du dix-septième siècle, et particulièrement après le complot des poudres visant à détruire le Parlement du royaume le 5 novembre 1605 . La dimension politique de la diatribe se cristallise autour de la figure du jésuite, agent du pape et ennemi naturel du royaume d'Angleterre et de son Église ${ }^{34}$. Cependant, dans le contexte agité du dix-septième siècle, l'anti-jésuitisme est loin d'être l'apanage des protestants, et on le retrouve au sein même des communautés catholiques : ainsi voit-on se développer en France, depuis la publication des textes monarchomaques du jésuite Juan de Mariana, une controverse dont les motifs sont en tous points similaires. Un mouvement hostile à la Compagnie de Jésus se développe donc à cette époque dans l'Église catholique elle-même, surtout sous le pontificat d'Urbain VIII (1623-44), pape sensible

32. Thomas Bell, The Anatomie of Popish Tyrannie, Londres, 1603, p. 8.

33. James Thomas, The Jesuits Downefall, Threatened against them by the Secular Priests, for their Wicked Lives, Accursed Manners, Hereticall Doctrine, and more then Machiavellian Policie, Oxford, 1612, avant-propos.

34. Parmi les publications mentionnées plus haut (note 5), voir par exemple Peter Lake, « Anti-Popery : the Structure of a Prejudice », in Richard Cust et Ann Hughes (dir.), Conflict in Early Stuart England; Peter Lake et Michael Questier, The Antichrist's Lewd Hat ; Arthur Marotti (dir.), Religious Ideology and Cultural Fantasy et Raymond Tumbleson, Catholicism in the English Protestant Imagination. 
aux plaintes des prêtres en lutte contre les ignaciens pour obtenir la direction des familles catholiques de la minorité récusante en Angleterre.

Ainsi, les polémistes protestants trouvent-ils un soutien inespéré quand le clergé séculier produit lui aussi des libelles anti-jésuites. Mary Ward se trouve prise sous les feux croisés de ce conflit. Si les prêtres rejettent une Compagnie de Jésus au féminin comme une absurdité, c'est pourtant bien en tant que « jésuitesses » qu'ils attaquent les Dames anglaises. Dans leur combat contre les jésuites et leurs imitatrices, les séculiers reprennent ainsi à leur compte les arguments typiques et codifiés des libellistes protestants. La diatribe aime se nourrir de détails qui font sensation, se plaisant à accuser les jésuites de graves débordements sexuels. John Baxter, anti-jésuite de renom, tonne contre les missionnaires : " ne font-ils pas tout pour séduire les pauvres femmes ${ }^{35}$ ? . Un autre pamphlet rapporte l'anecdote d'une jeune femme dont on découvre la grossesse quelques jours avant le mariage; elle finira par avouer que le père de l'enfant est un jésuite ${ }^{36}$. On va jusqu'à prétendre que les jésuites distribuent généreusement des dispenses à celles qui, enceintes, consentent à subir un avortement ${ }^{37}$.

Le clergé séculier attaque donc l'Ordre de Mary Ward par le même biais du scandale sexuel, s'appropriant ainsi l'une des armes de prédilection des polémistes protestants qui associent l'activité missionnaire à la débauche et dénoncent les jésuites comme les plus perfides des séducteurs, corrompant à l'envi les femmes qu'ils côtoient. C'est ainsi que dans les années 1620, les Dames anglaises sont accusées de relations charnelles avec leurs confesseurs jésuites ; l'une d'entre elles est même soupçonnée d'en attendre un enfant. On accuse Mary Ward d'inciter ses consœurs à user de leurs charmes

35. John Baxter, A Toile for Two-Legged Foxes, Londres, 1600, p.37.

36. William Fennor, Pluto his Travailes, or the Devils Pilgrimage to the Colledge of Jesuits, Londres, 1612, pp. 17-18 : « her belly [was] full of young bones, which [she] afterwards confessed were of the Jesuit's making ».

37. Anonyme, The Black Box of Roome Opened. From Whence are Revealed the Damnable Bloody Plots, practices and Behaviour of Iesuites, Priests, Papists, and Other Recusants in Generall, Londres, 1641, pp.16-17. 
pour parvenir à leurs fins, et d'avoir un jour déclaré que « [les Dames anglaises] feraient bien plaisir à Sa Sainteté et aux autres Cardinaux si elles s'exhibaient, la poitrine découverte, à la mode des courtisanes ${ }^{38}$. Dans son Informatio, l'archiprêtre William Harrison note que plusieurs de ces femmes sont réputées frivoles, et que leur mode de vie représente à lui seul un scandale pour l'Église. Le pamphlet du bénédictin Robert Sherwood accuse également les Dames anglaises de petite vertu et d'immoralité.

À ces accusations formulées par des clercs extérieurs à l'Institut, vient enfin s'ajouter un libelle anonyme paru en mars 1623 et connu sous l'intitulé Godfather's Information. Une section de ce pamphlet contient les dix-neuf déclarations de l'une des premières consœurs de Mary Ward, Mary Alcock, qui avait quitté l'Institut quelques années plus tôt et qui s'attache à le discréditer en attaquant personnellement sa fondatrice. Ce document outrancier et souvent invraisemblable n'offre guère de crédibilité. Néanmoins, par ses excès, il témoigne du climat particulier à cette période, tant il semble moins préoccupé de véracité que de sensationnel. Selon Mary Alcock, la population locale prendrait les établissements clandestins des Dames anglaises en mission à Londres pour des maisons closes ${ }^{39}$, et serait confortée dans cette idée par la conduite dissolue des Dames anglaises, qu'elle croirait être des prostituées. Ce témoignage suggère les mœurs des salons libertins plus que celles du couvent, évoquant des gorges découvertes et des robes aussi splendides qu'extravagantes, rehaussées de collerettes jaune vif pour lesquelles la fondatrice semble avoir une prédilection toute particulière. Et le rapport de s'insurger :

Une fois, [Mary Ward] habilla sa propre sœur Barbara Ward, d'une robe de taffetas de couleur vive taillée selon la dernière mode; ainsi affublée d'une collerette d'un jaune profond, la poitrine dénudée jusqu'au corset, elle l'envoya avec une seule compagne habillée de la

38. ABC, $\mathrm{C} 1$, Francesco Ingoli, Compendium of the Process made by the Nuncio of Cologne against the Jesuitesses, f.312.

39. ADW, vol. 17 (1623-24), ff.59-62, Godfather's Information about the Jesuitesses. Voir f.60, item 12 : « elles passaient pour des courtisanes et on les suspectait d'être des prostituées. » 
même façon, passer la nuit dans une auberge pour y convertir des âmes (selon ses dires); mais on n'a jamais entendu parler jusqu'ici de conversion des âmes par de tels moyens ${ }^{40}$.

Ornements évocateurs des mœurs sulfureuses de celles qui les portent, ces collerettes de couleur jaune avaient été lancées dans les milieux précieux de la société londonienne par une certaine Mrs Turner, criminelle, alchimiste et magicienne notoire, dont les filtres, onguents et autres potions réputées diaboliques lui vaudront la pendaison ${ }^{41}$. Mary Alcock souligne là les implications latentes de toute association, ne serait-ce que par le vêtement et la couleur, à Satan. La symbolique compare les Dames anglaises à des débauchées, séduites d'abord par la malice de la fondatrice elle-même et s'apprêtant à corrompre leurs victimes à leur tour.

Bien entendu, la luxure n'est pas le seul vice reproché aux Dames anglaises, que l'on accuse des sept péchés capitaux. Le témoignage de Mary Alcock insiste particulièrement sur la gourmandise de Mary Ward pour qui le moindre événement serait prétexte à de somptueux banquets; elle taxe aussi ses anciennes consœurs de paresse, leur indolence n'ayant rien de l'industrie nécessaire à l'apostolat. Enfin, dans huit de ses dix-neuf accusations, Mary Alcock souligne l'amour démesuré des Dames anglaises pour le luxe et l'argent. Or, ces reproches constituent eux aussi les bases de la diatribe contre les jésuites décrits comme sensuels, alliant la luxure à la paresse et à la gourmandise. L'accent est mis sur leur cupidité : citons par exemple le libelle d'Henry Compton, censé reproduire les instructions données aux jeunes jésuites en mission en Angleterre et mettant en exergue leur soif de pouvoir et de richesses. L'auteur assure qu'on enseigne aux postulants « comment se procurer la confiance de riches veuves », ou « comment monopoliser le contrôle des veuves et disposer librement de leurs richesses », chacune des

40. Ibid., item 14, f.61.

41. Victor MacLure, She Stands Accused, Being a Series of Accounts of the Lives and Deeds of Notorious Women, Murderesses, Cheats, Cozeners, on whom Justice was Executed, and of others who, Accused of Crimes, were Acquitted at least in Law; Drawn from Authenticated Source, Londres, 1935, p. 56. 
instructions visant à accroître la fortune de la Compagnie, aux dépens même de la morale ${ }^{42}$.

Selon les pamphlétaires anti-jésuites, les ignaciens ne reculent devant aucun stratagème pour piéger leurs victimes : le champ lexical du prédateur et de sa proie est décliné dans de nombreux ouvrages, tels ceux de John Gee ou de Samuel Harsnett, où les auteurs décrivent les spectaculaires mises en scènes auxquelles les missionnaires auraient recours pour amener les crédules à se convertir ${ }^{43}$. On y trouve de fausses possessions démoniaques, donnant lieux à des simulacres d'exorcisme tendant à prouver le pouvoir des clercs, ainsi que des apparitions de spectres terrifiants, selon des procédés scéniques utilisés au théâtre. Or, on dit aussi des Dames anglaises qu'elles « feignent des révélations pour confirmer leur Institut [...]». Selon Francesco Ingoli, le secrétaire de Propaganda Fide, ce sont des manipulatrices, des séductrices menant tant leurs adeptes que l'Église à la destruction. À maintes reprises, il les accuse : « [Les Dames anglaises] séduisent les jeunes filles en les attirant dans leur Compagnie ${ }^{44}$; «par la séduction, elles ont convaincu diverses jeunes femmes d'entrer dans leur congrégation en leur faisant croire qu'elle était officiellement approuvée ${ }^{45}$. Le thème de la séduction, que l'on retrouve si souvent sous la plume des auteurs anti-jésuites ${ }^{46}$, est repris ici par le clergé séculier pour condamner les « jésuitesses » comme autant d'ensorceleuses qui détournent les âmes du droit

42. Henry Compton, The Jesuites' Intrigues, with the Private Instructions of that Society to their Emissaries, Londres, 1669, pp. 35 et 38.

43. Voir entre autres John Gee, The Foot out of the Snare. With a Detection of Sundry Late Practices and Impostures of the Priests and Iesuites In England, Londres, 1624 et New Shreds of the Old Snare, Londres, 1624, ainsi que Samuel Harsnett, A Declaration of Egregious Popish Impostures to Withdraw the Harts of her Maiesties Subjects from their Allegeance and from the Truth of Christian Religion Professed in England, under the Pretence of Casting out Devils, Londres, 1603.

44. ABC, C1, Letters against the Jesuitesses, 1630, f. 312.

45. $\mathrm{ABC}, \mathrm{C} 1$, Francesco Ingoli, Compendium of the Process made by the Nuncio of Cologne against the Jesuitesses, f. 311.

46. Laurence Lux-Sterritt, « La séduction au service de la perfidie : représentations des missionnaires de la Contre-Réforme en Angleterre », à paraître. 
chemin et fragilisent l'ordre établi. L'archiprêtre William Harrison les accuse de profiter de la vulnérabilité des Anglaises venues sur le Continent pour y être éduquées dans la foi catholique avant de rentrer au pays ; elles les séduisent, écrit-il, et les détournent de leur projet initial pour les persuader de se joindre à elles. Selon son Informatio, les couvents de Louvain et Gravelines sont floués par les Dames anglaises, qui les privent de leurs nouvelles novices, mettant ainsi en danger la survie des monastères traditionnels. Mary Alcock souligne également que l'Institut favorise la Compagnie de Jésus aux dépens des autres Ordres, déclarant que Mary Ward et ses consœurs ne respectent que les jésuites et dénigrent tous les autres clercs ${ }^{47}$.

Nous le voyons bien, si les séculiers prétendent qu'il est impossible d'envisager une Compagnie de Jésus pour femmes, ils attaquent pourtant les Dames anglaises, les « jésuitesses », comme on attaque traditionnellement les jésuites. Car même si le clergé ne reconnaît pas à l'Institut le droit d'exister, il est cependant bien là, et ses Dames anglaises s'emploient chaque jour à promouvoir l'idéal ignacien et à confier leurs adeptes aux jésuites. Les rapports de Robert Sherwood OSB et de Matthew Kellison prétendent d'ailleurs que le jésuite Roger Lee, ancien père spirituel de Mary Ward, serait le véritable fondateur de l'Institut. Pour le bénédictin, la présence des Dames anglaises en Angleterre n'a d'autre but que de préparer le terrain des jésuites, à qui elles confient la direction spirituelle de familles entières. Les disciples de Mary Ward représentent donc un double danger pour l'équilibre de l'Église, d'une part par les innovations qu'elles proposent et qui bafouent des siècles de tradition, d'autre part par leur dévouement total à la Compagnie de Jésus pour la promotion de laquelle elles travaillent sans relâche au détriment de tous les autres Ordres.

L'Institut de Mary Ward sera ainsi victime de la complexité du contexte religieux dans lequel il est né. Dans l'Église post-tridentine, où l'historienne Elizabeth Rapley remarque « une dichotomie masculin-féminin de plus en plus marquée, un antiféminisme agressif,

47. ADW, vol. 17 (1623-24), ff.59-62, Godfather's Information about the Jesuitesses, f.59, item 1. 
un mouvement irrésistible vers le patriarcat ${ }^{48}$, l'Institut des Dames anglaises est condamné comme un nouvel Ordre féminin jugé inacceptable et absurde. Mais cette simple opposition, rencontrée par ailleurs par nombre de nouveaux mouvements féminins au XVII siècle, se double ici d'un écueil de nature plus politique : pour l'Église, déchirée par la tourmente qui oppose le clergé séculier aux jésuites, Mary Ward est un témoignage vivant de l'ascendant qu'exerce la Compagnie de Jésus sur les milieux dévots de l'époque. Outre son mépris des traditions monastiques, la mission de l'Institut donne un avantage non négligeable aux jésuites, leur confiant la direction de familles nouvellement converties. Dans le contexte des années 1620, une telle congrégation ne saurait qu'encourir la réprobation des adversaires de la Compagnie de Jésus.

C'est pourquoi, en septembre 1630, l'Église charge le nonce Pierluigi Carafa d'interroger sept des membres de l'Institut, dans leur communauté de Liège ${ }^{49}$. La transcription de l'audience est envoyée à Rome et étudiée par Francesco Ingoli, secrétaire de la Propaganda Fide, ouvertement hostile aux jésuites ; dans son rapport, il condamne l'Institut et demande que soient prises des mesures punitives. À l'issue des délibérations de la Congrégation le 21 novembre 1630, Urbain VIII remet l'affaire entre les mains de l'Inquisition, qui décide d'emprisonner Mary Ward en février 1631 à Munich. Le couperet tombe quelques temps plus tard, quand Urbain VIII déclare la suppression des dites « jésuitesses » dans sa Bulle Pastoralis Romani Pontificis. Mary Ward est déclarée hérétique, son Institut réduit au rang de secte bafouant tant la décence féminine que les prérogatives du clergé : le souverain pontife veut éradiquer «ces plantes nuisibles à l'Église de Dieu». Il ordonne de les « arracher jusqu'aux racines, et pour les empêcher d'abonder, de s'étendre et de s'accroître, les détruire entièrement ${ }^{50}$ car, selon lui, les Dames anglaises menacent l'ordre établi bien plus qu'elles ne le servent.

48. Elizabeth Rapley, The Dévotes : Women and Church in Seventeenthcentury France, Kingston, Ont., 1990, p. 3.

49. Immolata Wetter, Mary Ward, pp. 38-59.

50. Bibliothèque nationale de France, $\mathrm{Z}$ Thoisy, 312, Pièces relatives à la suppression de l'ordre par Urbain VIII en 1631. Traduction française de la bulle papale Pastoralis Romani Pontificis. 
Après sa libération, Mary Ward se retire en Angleterre, où elle continue à œuvrer pour la communauté récusante avec quelques-unes de ses consœurs, à titre privé ; elle meurt dans le Yorkshire en 1645. L'Institut survit pourtant grâce à l'électeur de Bavière Maximilien I (1573-1651), qui permet aux Dames anglaises installées à Munich de continuer à faire la classe, à condition bien sûr de ne plus prétendre être un Ordre religieux ${ }^{51}$. C'est donc de Munich que partira leur effort renouvelé pour obtenir l'approbation papale. Ce nouvel Institut se concentre sur l'enseignement des filles, garde le cloître et ne revendique pas de vocation missionnaire ; il n'a, officiellement, plus rien en commun avec celui fondé par Mary Ward et supprimé en 1631. En 1749, Benoît XIV dans sa Bulle Quamvis Justo le reconnaît sous le nom d'Institut de la Bienheureuse Vierge Marie. Ce n'est que le 7 juin 2003 (près de quatre siècles après que Mary Ward déclare avoir reçu l'ordre divin, «Take the same of the Society »), que l'Église permettra enfin à la vocation de la fondatrice d'exister telle qu'elle l'aurait souhaité, en adaptant les règles d'Ignace de Loyola pour une congrégation de femmes à qui l'on octroie enfin le droit de porter le nom de Congrégation de Jésus ${ }^{52}$.

\section{LERMA}

Université d'Aix-Marseille I

29 avenue Robert Schuman

13620 Aix-en-Provence cedex 1

51. Voir Henriette Peters, Mary Ward, pp. 594-97.

52. Je tiens à remercier $\mathbf{M}^{\text {me }}$ Sophie Houdard (Université de Paris III Sorbonne Nouvelle) pour ses précieuses remarques à propos de cet article. 